\title{
Linx
}

Revue des linguistes de l'université Paris X Nanterre

$59 \mid 2008$

Les conjonctions en diachronie : parcours sémantiques

\section{Les conjonctions de causalité et leur grammaticalisation}

\section{Gerda Haßler}

\section{OpenEdition}

\section{Journals}

Édition électronique

URL : http://journals.openedition.org/linx/628

DOI : $10.4000 /$ linx.628

ISSN : 2118-9692

Éditeur

Presses universitaires de Paris Nanterre

Édition imprimée

Date de publication : 1 décembre 2008

Pagination : 95-114

ISSN : 0246-8743

Référence électronique

Gerda Haßler, « Les conjonctions de causalité et leur grammaticalisation », Linx [En ligne], 59 | 2008, mis en ligne le 01 janvier 2012, consulté le 10 décembre 2020. URL : http://journals.openedition.org/ linx/628; DOI : https://doi.org/10.4000/linx.628 


\title{
Les conjonctions de causalité et leur grammaticalisation
}

\author{
Gerda Haßler \\ Universität Potsdam
}

Dans cet article, nous étudierons d'abord la nature du lien causal entre deux propositions pour regarder ensuite les pistes de grammaticalisation des marqueurs subordonnants de la causalité. Nous montrerons les bases cognitives de la grammaticalisation des locutions conjonctives de causalité et nous vérifierons le résultat de cette grammaticalisation des connecteurs parce que et puisque dans les textes de la Base du Moyen Français. Le changement sémantique des locutions françaises sera comparé à celui d'autres langues. Finalement, nous exposerons des conclusions sur la méthode de description de ces changements en termes de grammaticalisation.

\section{L'expression d'une relation causale entre deux propositions dans les langues romanes anciennes}

Même si elles apparaissent comme syntaxiquement indépendantes, deux propositions qui se suivent dans le discours ne sont en général pas ressenties comme complètement indépendantes, mais comme liées par quelque lien sémantique, fût-ce par référence à un contexte plus large ou à la situation. La relation sémantique qui existe entre les deux phrases peut être considérée comme un lien de causalité dans lequel (1a) représente la cause de (1b) :

1a) Marie est fatiguée.

1b) Elle se coucha.

Les deux propositions sont logiquement l'antécédent et le conséquent, il y a alors une relation sémantique d'interdépendance. Robert de Dardel (1983: 16) emploie 
même les termes de régissante et de dépendante, étant entendu qu'il n’y a pas d'isomorphisme entre les deux couples terminologiques. Dans l'exemple (1), la première proposition peut être une dépendante causale, dans le sens étroit du terme, de la seconde, et la seconde peut être une dépendante consécutive de la première. Les traits sémantiques présents dans ces deux propositions donnent la base de cette relation de causalité qui s'appuie en outre sur des traits syntaxiques, tels que la position des termes et la nature des propositions. Les propositions qui sont liées entre elles par des traits sémantiques et des traits complémentaires à l'exclusion des traits syntaxiques de la coordination et de la subordination forment une unité interpropositionnelle que caractérise sur le plan syntaxique, la juxtaposition (Dardel 1983 :17).

Le premier pas vers une expression explicite du lien causal entre deux propositions est l'usage d'une conjonction de coordination introduisant une proposition qui explique ou justifie ce qui vient d'être énoncé. En français, on trouve aujourd'hui, la conjonction $\operatorname{car}^{1}$ qui peut être utilisée après ponctuation faible (2a) ou sans ponctuation (2b) ainsi qu'après ponctuation forte ((2b), (2c) et (2d)) :

2a) Grâce au médecin, l'existence prit un rythme normal. Cette espèce de confort n'influençait guère les enfants, car ils avaient le leur et qui n'était pas de ce monde. (Cocteau, Les Enfants terribles, 1929 : 53).

2b) Félicien, en revanche, s'était installé à califourchon sur une chaise, s'essuyait les mains à son tablier, un bout de chiffon à l'un ou l'autre doigt car il s'était coupé (Cendrars, Bourlinguer, 1948 : 283).

2c) Et je sortis avec une grande soif. Car le goût passionné des mauvaises lectures engendre un besoin proportionnel du grand air et des rafraîchissants. (Baudelaire, Petits poèmes en prose, Assommons les pauvres, 1867 : 216).

2d) Et je suis sûr que c'était un mort; car il a disparu tout d'un coup en remuant son doigt comme pour me faire signe de venir. (Loti, Mon frère Yves, $1883: 87)$.

2e) [...] telle fut la dernière vision que Marie eut de ce monde. Car tout aussitôt une atroce déchirure se fit à son côté (...) et... et Joseph, les jambes écartées pour avoir toute sa force, sentait les chairs de Marie s'ouvrir sous le couteau. [Jouve, La Scène capitale, $1935: 21] .^{2}$

\footnotetext{
${ }^{1}$ Sur car dans la diachronie du français of. Fagard \& Degand 2008.

${ }^{2}$ Les références bibliographiques de ces exemples, ainsi que ces en français moderne dans l'article, sont toutes prises du Trésor de la langue Française Informatisée (http://atilf.atilf.fr/tlf.htm) et n’apparaissent pas dans la bibliographie.
} 
Ces exemples montrent que la conjonction car rend explicite une relation causale qui existe déjà entre les deux propositions. Il peut être préférable d'employer cette conjonction de coordination pour faciliter le travail cognitif de la reconnaissance du lien causal. À part cela, car n'a aucune fonction dans la phrase et il peut s'ajouter sans modifier la structure syntaxique ou le degré de la liaison entre les deux propositions qui s'exprime, en langue écrite, par la ponctuation.

La conjonction de coordination car dérive directement du latin quare $(<q u \vec{a}$ re) 'c'est pourquoi' qui a donné la forme KA en roman commun tardif et qui était le connecteur causal le plus utilisé (Dardel 1983 : 171). Les exemples 3a) à 3d), qui sont tirés de textes en langues romanes, montrent que KA s'utilisait comme un connecteur de coordination qui rend explicite la liaison causale entre les propositions en question et qui n'a pas d'influence sur la structure syntaxique ${ }^{3}$ :

3a) esp. Sospiró moi Cid, ca mucho avie grandes cuidados.

[Mon Cid soupira, car il avait grande peine] (Cid, Menéndez Pidal 19541956 : VI, 1025)

3b) port. Mas ella, por su vemtujra, casou-sse melhor que nemhuma das outras, ca se pagou della elrrey de Framça e filhou-a por molher.

[Mais elle, pour son bonheur, se maria mieux qu'aucune des autres, car le roi de France s'éprit d'elle et la prit pour femme] (Livro de linhagens, Nunes $1970: 13$ )

3c) it. Non è miravegla se l'omo no vole succurrer a l'altro in necessitade. Ka per le peccade nostre la fede à perduta in terra, \& no se trova la veritade leve mente in questo mundo.

[Rien d'étonnant si l'homme refuse desecourir son prochain, car à cause nos péchés la fidélité a disparu de la terre et la vérité ne se trouve pas facilement dans ce monde] (Guido Fava, Gemma Purpurea. Lazzeri 1954 : 420).

3d) roum. Şi ca nū văduru de slava lūmirïei aceei, de măînră priî̀nnseră-me ceřa ce erà cū meînre.

[Et comme je n'y voyais pas, à cause de l'éclat de cette lumière, ceux qui étaient avec moi me prirent par la main] (Codicele Voronețean, Sbiera $1885: 40)$

En dehors des possibilités de la juxtaposition de deux propositions liées par un lien logique de causalité et de la coordination rendue explicite par une conjonction de coordination, la cause peut être formulée dans une proposition subordonnée. Cette construction est d'une complexité cognitive plus grande parce qu'elle implique l'intégration de la proposition causale dans la phrase matrice :

\footnotetext{
${ }^{3}$ L'origine étymologique de / ka/, dans ces langues, n'est pas l'objet de la présente étude.
} 


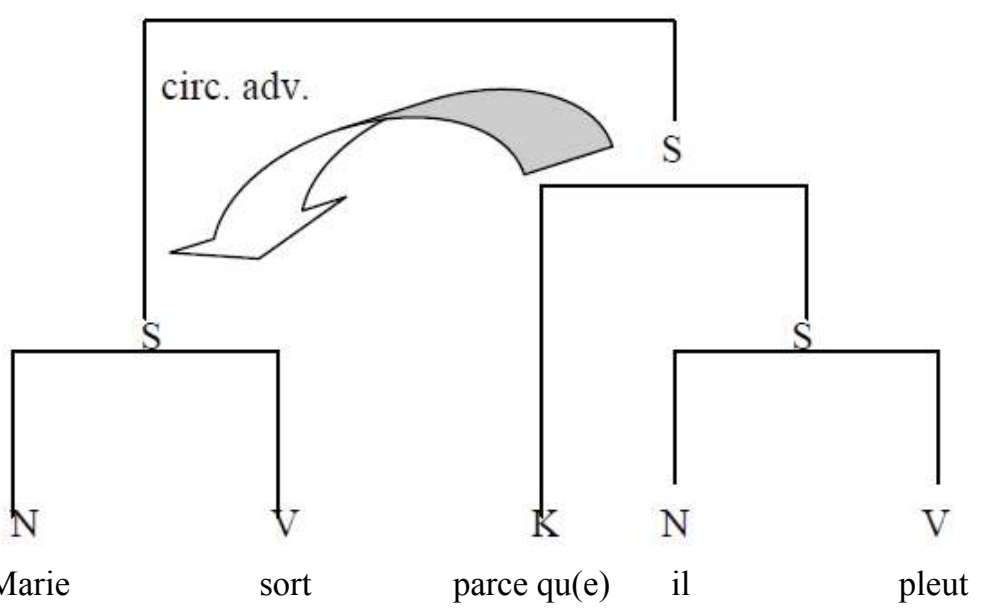

La liaison causale établie par le connecteur subordonnant peut contredire la relation entre une cause et une conséquence telle qu'elle est établie par l'expérience des locuteurs. Dans l'exemple (4) on ne s'attendrait pas à ce que la pluie soit une raison pour sortir. C'est-à-dire que le connecteur, dans ce cas, n'opère pas seulement une intégration de la proposition causale dans la phrase, mais il porte aussi les éléments sémantiques les plus importants.

On trouve, déjà, dans les premiers textes en langues romanes, des connecteurs subordonnants. Selon Dardel (1983: 132) quomodo, qui introduit en roman commun tardif une subordonnée causale antéposée à la principale, a exprimé la cause évidente, fonction qu'on lui retrouve encore dans les parlers romans modernes :

5) Comme il est malade, il ne peut pas venir à la réunion.

La proposition causale introduite par comme se trouve toujours en position thématique de la phrase et elle exprime des informations mentionnées avant ou généralement connues. Dans les exemples suivants, les conjonctions dérivées de quomodo introduisent une proposition causale au début de la phrase :

6a) esp. Commo sodes muy bueno, tener la edes sin arth.

[comme vous êtes très brave, vous la [la bannière] porterez loyalement] (Cid, Menéndez Pidal 1954-1956 : 1052)

6b) cat. Con algu no sabes de la novia tot hom stech fort meravellat. 
[Comme personne n'avait den ouvelles de la fiancée, chacun fut fort surpris] (Par 1923 : 365)

6c) anc. fr. «Dex», dist la damme, «que ferai ? com fui grosse, / De ceste gent ne cuit que nus estorde.»

["Dieu », dit la dame, "que ferai-je ? Comme je fus grosse, je pense que personne de ces gens-ci ne s'en sortira. »] (Jourdain de Blaye, Dembrovski $1969: 77)$

Quomodo est un connecteur qui, ne serait-ce que par le fait d'introduire une subordonnée antéposée, n'exprime jamais une justification après coup, tandis qu'en roman commun tardif, le connecteur KE prend la fonction de cause non évidente. Cela ressort également de passages où, en français moderne, on introduit une causale par que en réponse à une question du type Pourquoi:

7) M. DUverdier. Et pourquoi diable voulez-vous que je vous trompe? M. BÉTASSIER. C'est que vous m'avez déjà trompé plusieurs fois. (Louis CARMONTELLE, L'Uniforme de campagne, 1781 : 370-37.)

8) Le MARquis. Pourquoi donc ? Bernardy. C'est que j'ai rencontré un homme qui courait aussi bien que moi, avec une béquille à la main ; c'est sûrement votre voleur. (Louis CARMONTELLE, L'Uniforme de campagne, $1781: 520)$

Cependant, dans le cas de KE, seules les constructions causales ressortissant à la justification après coup, liée à une principale impérative, interrogative ou exclamative, restent vivantes. Dans les phrases c), KE introduit une proposition rhématique qui porte tout le poids informatif et que la conjonction transforme en proposition causale :

9a) occ. E martha ua dire: « senher, el flaira, que .IIII. iorns a istat mortz ». [Marthe répondit : "Seigneur, il sent déjà ; car il est mort depuis quatre jours »] (Evvangile selon saint Jean 11.39, Wollenberg 1868 : 11.39).

9b) anc.fr. Super lis pez ne pod ester, / Que toz los at il condemnez.

[Il ne peut se tenir sur les pieds, car il les a tout endommagés] (Vie de saint Léger, Linskill 1937 : 169)

9c) it. li nostri patri male pinzaru, / ke contra Deo revillaru ;

[Nos pères furent mal avisés, car ils s'insurgèrent contre Dieu] (Elegia giudeo-italiana, Lazzeri 1954 : 184)

Après une principale simplement déclarative, où la relation de causalité est peut-être moins marquée et le connecteur senti comme trop peu explicite, KE est 
remplacé dès le roman commun tardif par PRO-KE qui permet l'antéposition de la subordonnée (Dardel 1983 : 132) :

10a) esp. mas por que me vo de tierra, dovos çinquaenta marcos [mais comme je quitte le pays, je vous donne cinquante marcs] (Cid, Menéndez Pidal 1954-1956 : 1034)

10b) cat. Havia vergonya de descobrir-li lo seu cor perquè molt lo temia e amava.

[Il avait honte de lui découvrir son coeur, car il le respectait et l'aimait beaucoup.] (Ramón LLull, Moll 1952 : 394)

10c) it. Amor m'auzide. - Perché ? - Per ch'io amo.

[L'amour me tue. - Pourquoi? - Parce que j’aime.] (Ser Iacopo da Leona, sonnet 2, vers 1, Massèra $1920:$ I, 32)

\section{La causalité en tant que catégorie cognitive}

Avant d'étudier le développement des connecteurs causaux en français, nous étudierons la catégorie cognitive de la causalité. Le but de cette analyse est l'établissement de relations formelles que nous utiliserons dans l'étude des connecteurs causaux en français 4 .

La causalité est un concept épistémologique qui consiste à organiser systématiquement les faits empiriques et à leur donner sens. Selon cette définition constructiviste, la causalité formalise le fondement même de toute démarche scientifique. La causalité est donc le lien construit entre une cause (ou un ensemble de causes) et une conséquence.

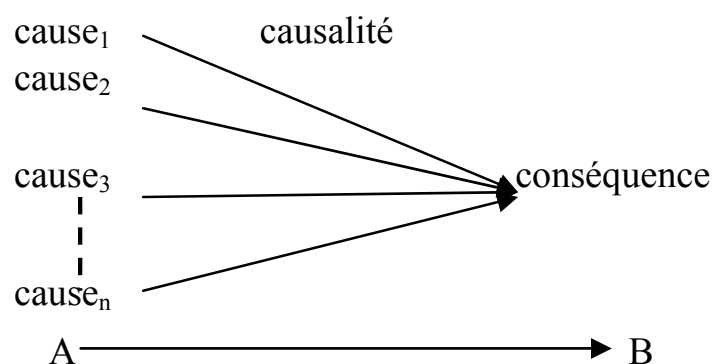

La version la plus épurée de la causalité est dite linéaire (A, donc $B$, avec éventuellement des étapes causales intermédiaires) ; elle suppose une antériorité, même infime, de la cause sur l'effet. De ce fait, elle a partie liée avec la succession de la

\footnotetext{
${ }^{4}$ Sur les moyens linguistiques qui expriment la cause en ancien français, cf. Bertin (1997).
} 
conséquence à la cause et se moule aisément dans les modes d'écriture linéaire fondés justement sur la succession. Des constructions causales peuvent rester linéaires tout en introduisant des causalités multiples (A et/ou A', donc B); mais généralement la multiplicité des causes introduit des effets rétroactifs.

Cette relation entre le temps et la causalité rend possible l'usage de connectifs temporels pour l'expression de la causalité. Selon le principe post hoc ergo propter hoc, le subordonnant temporel de postériorité ou de point de départ depuis devient causal, il en est ainsi avec since en anglais, sintemalen en allemand et puisque en français.

\section{postériorité}

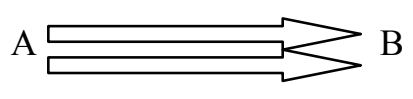

causalité

Le subordonnant temporel de simultanéité peut aussi devenir causal. Ainsi, des mots dérivés de l'étymon QUANDO s'utilisent aussi comme introducteur d'une proposition causale :

11a) anc. fr. Or irez vos certes, quant jol cumant.

[Vous irez certainement maintenant, puisque je le commande] (Roland, Moignet 1969 : 47)

11b) esp. Grado a Dios, aquel que está en alto, / quando tal batalla avemos arrancado

[Dieu sois loué, celui qui est aux cieux, puisque nous avons triomphé en une telle bataille !] (Cid, Menéndez Pidal 1954-1956 : 1056)

C'est aussi le cas de la conjonction causale weil en allemand qui dérive de la locution temporelle thiu (b)wìla sō qui signifiait au 11e siècle 'pendant le temps que'. En moyen haut allemand, die wile (aussi al die wile) avait le sens de 'pendant que' et, par la chute de l'élément connectif so, la locution conjonctive temporelle die wile 'pendant' s'est développée pour se transformer, au $15^{\mathrm{e}}$ siècle, en la conjonction simple weil (Pfeifer 1989 : 1953). Jusqu'au 18 siècle, cette conjonction s'utilisait surtout dans le sens temporel, mais on trouve l'usage causal dès le $14^{\mathrm{e}}$ siècle, dans des contextes où la fonction temporelle coïncide avec la fonction causale. Les conjonctions néerlandaise (wijl) et anglaise (while) ont parcouru le même chemin.

En allemand, on trouve un subordonnant local (da) qui est devenu temporel et, par la suite, a développé une signification causale introduisant la présupposition explicite d'une conclusion :

12) Da das Pflaster nass ist, hat es offenbar heut Nacht geregnet. [Comme le pavé est humide, il a dû pleuvoir cette nuit.] 
Dans ces exemples, la conjonction marque la présence du fait considéré comme cause en même temps que le contenu de l'autre proposition. Cette co-présence permet d'établir une relation de causalité entre $\mathrm{A}$ et $\mathrm{B}$ dans laquelle $\mathrm{B}$ est regardé comme la conséquence inévitable de A :

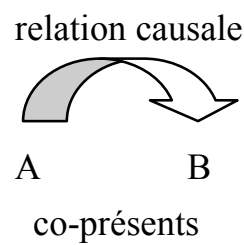

Comme nous l'avons vu, la causalité est une catégorie épistémique qui peut être exprimée par des subordonnants qui avaient primitivement d'autres fonctions. C'est le cas des quatre subordonnants hérités du latin par le roman (comme, quand, que, si) qui peuvent introduire une proposition causale. Comme qui renvoyait d'abord à une certaine manière (lat. quomodo 'de la manière que') peut introduire une subordonnée causale antéposée à la principale, dans le cas de quand (lat. quando), la co-présence temporelle de deux faits peut être réinterprétée comme cause et conséquence. Le connecteur que peut apparaître dans des constructions causales ressortissant à la justification après coup et, au cours du temps, il fut remplacé dès le roman commun tardif par PRO-KE qui permet son usage dans d'autres positions. Même la conjonction conditionnelle si peut être réinterprétée comme un marqueur de causalité si la proposition $p$ exprime une éventualité qui justifie causalement la proposition $q$ :

13) L'Autriche ne me fera pas la guerre. [Si elle me la fait = p], [j'ai 150000 hommes en Allemagne, et autant sur le Rhin, et 400000 Allemands pour lui répondre $=$ q] (NAPOLÉON Ir, Lettres Joseph., $1809: 167)$

Les traits universels de la causalité se manifestent par leur présence constante dans toutes les langues ou par leur apparition possible dans toutes les langues connues. C'est le cas de l'amplification par métonymie, qui produit des subordonnants causaux à partir de subordonnants locaux, temporels, modaux et conditionnels.

Une réflexion sur l'évolution sémantique permet de déterminer pour l'amplification métonymique, sinon des lois, du moins des tendances en ce qui concerne les fonctions exprimées :

a) Le subordonnant local devient temporel (allemand da)

b) Le subordonnant temporel marquant la postériorité ou le point de départ 'depuis' devient causal (anglais since, allemand sintemalen, français puisque).

c) Le subordonnant temporel de simultanéité devient causal (allemand weit)

d) Le subordonnant comparatif d'égalité devient causal (anglais as, français comme) 
L'amplification métonymique fonctionne selon le principe que, dans certains contextes, une unité ayant la fonction A se laisse aussi interpréter comme ayant la fonction B, de sorte que l'unité de départ finit par se diviser en deux unités ayant le même ordonnant. Cette amplification métonymique exclut par conséquent, dans son processus même, tout changement des autres traits, par exemple sous la forme de modifications des règles syntaxiques.

\section{Les connecteurs parce que et puisque et leur parcours de grammaticalisation en français}

Le fait que tous les subordonnants hérités du latin peuvent être utilisés comme connecteurs causaux montre l'importance cognitive de la causalité : les hommes sont enclins à interpréter des relations modales, temporelles et conditionnelles comme causales. D'autre part, ce fait montre qu'il n'y avait pas, en ancien français, de subordonnant central pour exprimer la relation causale. Cela pourrait être considéré comme une raison du développement de plusieurs locutions conjonctives qui servent à ce propos (sur les connecteurs principaux of. Bertin 1997, Chapitre II).

La principale source pour le développement des connecteurs français est la combinaison d'une préposition et du morphème complémenteur que, avec entre les deux parfois le démonstratif neutre cataphorique ce. L'importance quantitative de ce processus a été montrée par Bat Zeev Shyldkrot et Kemmer (1988). Dès l'ancien français, on trouvait les locutions conjonctives par ce que, por ce que, puis que, des ce que, après ce que, etc., qui sont à l'origine des locutions parce que, pour que, puisque, après que, avant que, depuis que, dès que, jusqu'à ce que, selon que du français moderne.

Les deux connecteurs causaux parce que et puisque ont subi un changement sémantique menant à l'expression de la relation logique de causalité.

\subsection{Parce que}

Parce que apparait vers 1200 sous la forme par ce ke, qui s'analyse comme une locution conjonctive composée de la préposition par, du démonstratif ce et de la conjonction que, la dernière représentant l'élément conjonctif le plus général. De par sa structure

$$
\operatorname{par}(<\text { lat. per })-c e(<\text { lat. hoc })-q u e(<\text { lat. quid })
$$

cette locution conjonctive représente, de manière analytique, l'expression de la cause 'par la chose/le fait que...'.

14) Je suis inquiet parce que je vous aime.

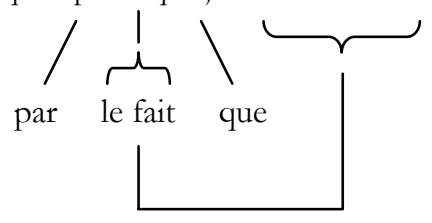


Du point de vue cognitif, le pronom ce dans cette locution représente une particularité par rapport aux autres langues romanes. Celles-ci continuent la forme PRO-KE du roman commun tardif qui élimine l'élément pronominal : espagnol porque, portugais porque, catalan perquè, italien perché. Dans ces langues, la conjonction causale de subordination correspond par sa forme au mot pourquoi. Mais, dans d'autre langues, on trouve des locutions conjonctives qui suivent le même modèle que le français par ce que. Le russe nотомy umo suit exactement le même principe, y compris la fusion entre no 'par' et томy 'ce'. Le connecteur anglais because suit un modèle similaire mais en omettant l'élément conjonctif 'que' : en moyen anglais la locution conjonctive était composée de deux parties : bi 'par' et cause 'raison'

La locution par ce que a remplacé la locution médiévale de même sens pour ce que présente vers 950 . Au $14^{\mathrm{e}}$ siècle, la soudure des deux premiers éléments produit la forme parce que. L'élément pronominal est pourtant toujours conçu comme tel, ce qu'on peut voir dans l'emploi direct de parce que avec ellipse de la proposition principale, en réponse à une question commençant par pourquoi (15). La locution est employée seule, de manière elliptique, pour exprimer un avis, un ordre péremptoire dont on ne peut ou ne veut donner les raisons (16) (Rey 2000 : 2568) :

15) Pourquoi? Dame! Parce que... c'est impossible, voilà tout. Je ne veux pas. (ZOLA, Nana, 1880, p. 1334).

16) «Nous devrions nous tutoyer, comme autrefois: voulez-vous?» «Non.» «Pourquoi?» «Parce que!» (FLAUBERT, Éduc. sent., t.2, 1869, p. 64).

Le caractère pronominal de ce dans parce que permet de l'utiliser comme un groupe nominal qui peut être utilisé elliptiquement en fonction nominative.

Dans la Base Frantext Moyen Français, nous avons trouvé plusieurs emplois pronominaux de $c$, avec lesquels par ce que se trouve en analogie 17):

17a) Et par ce avrez dame en qui compassee / Sera beauté qu'Amours scet preparer. (Alain CHARTIER Le Lay de Plaisance, c.1412, 153)

17b) Par ce transporte Dieu lez royaulmes de main en aultre. (Alain ChARTIER Le Liure de l'Espérance, c.1429-1430, 42)

17c) A occire adés, sanz tarder, Car, par ce, se cuida garder De la mauvaise destinee, Qui ainsi estoit destinee (Christine de PIZAN Le Livre de la Mutacion de Fortune, t.2, 1400-1403, 285-286)

17d) Car bien sot que son frere aroit, / Par ce, ayde et plus fort seroit, / Car de lui le regne laissier / Au bout de l'an, pour commencier/ A regner, n'ot il voulenté. (Christine de PIZAN Le Livre de la Mutacion de Fortune, t.2, 1400-1403, 301) 
17e) Cassandra, la sage clergece, / Fille au roy, par sa grant sagece,/Bien savoit qu'il devoit venir / Mal par ce, le temps avenir (Christine de PIZAN Le Livre de la Mutacion de Fortune, t.3, 1400-1403, 67)

Dans les phrases (17a) à (17e), le pronom ce remplace la dénomination d'une action, d'un fait, d'une chose ou d'une circonstance qui ne se trouve pas en relation causale avec le reste de la phrase, mais qui pourrait être plutôt interprété comme un instrument (dans le sens large) ou une voie pour atteindre quelque chose.

La même procédure s'utilise dans la locution conjonctive par ce que qui a la capacité syntaxique de subordonner une proposition qui explique, de manière prédicative, cette action, ce fait, cette chose ou cette circonstance. Cette explication ne peut pas être interprétée comme en premier lieu causale, comme on peut le voir dans les exemples suivants.

Dans l'exemple (18), la relation mutuelle de l'existence et de la connaissance de quelque chose est constatée, c'est une relation de fait et non en premier lieu une relation causale. Les choses n'existent que par la connaissance que Dieu en a, et la connaissance de l'homme dépend de leur existence. Cette relation factuelle pourrait s'interpréter secondairement comme causale :

18) Car ta science despent des choses que tu sçais de luy, et les choses qu'il sçait despendent de sa science. Elles sont par ce qu'il lez sçait, et tu les sçaiz par ce qu'elles sont. Leur mutation ne peult muer sa science, car sa science precede leur estre. (Alain CHARTIER Le Livre de l'Espérance, c.1429-1430, 160)

Dans l'exemple (19), la phrase introduite par par ce que donne une réponse à la question Comment?, ce qui peut être regardé comme un indice du fait qu'il s'agisse d'une manière et non d'une cause.

19) Esperance : Ton argument procede de ignorance.

Entendement : Comment?

Esperance : Par ce que tu ne congnoiz la difference et condition des qualités et des atributions des noms de Dieu. (Alain CHARTIER Le Livre de l'Espérance, c.1429-1430, 156)

Dans l'exemple (20), il s'agit d'un renvoi à un acte de parole qui ne peut pas être regardé comme la cause des actions dans le reste de la phrase et, dans (21) on décrit la nomination du roi comme une circonstance ou une présupposition.

20) Et doncques, par ce que vous dictes / Ce loz et pris que les chiefz ont / Ne vient mie par leurs merites, / Quant ce sont ceulx qui moins en font. (Alain CHARTIER Le Debat du Herault, du Vassault et du Villain, p. 1415, 428)

21) Car, par ce que roy fu nommé / En son regne, et tant renommé /L'enfent, pour celle occasion, /Le roy crut que l'avision, / Qu'il ot faite, 
fust avoirie. (Christine de PIZAN Le Livre de la Mutacion de Fortune, t.2, 1400-1403, 196)

Les phrases (18) à (21) montrent que le processus de grammaticalisation de la locution parce que comme conjonction causale n'était pas encore terminé au $15^{\mathrm{e}}$ siècle. Cela peut être démontré par l'écriture disjointe de par ce que et par les différentes interprétations du pronom ce qui peut signifier autre chose qu'une 'cause'.

Pour le siècle suivant, on trouve des données dans Frantext qui présentent une interprétation nettement causale de par ce que:

22a) Cancer est ainsin dit par ce que le chancre chemine en arriere. [...] Leo est ainsin nommé par ce que le Soleil ou moys d'aoust et ou signe de Leo est treschault, ardent et presque intollerable. Aussi le lyon est une beste moult chaude, et de ire importable. (Jehan THENAUD, Traité de poesie ou Traité de science poeticque, 1515-1519, p. 114)

22b) Cestuy surmonta les Indes, car la chaleur du Soleil rent les Indoys bons buveurs, ou par ce que en Inde le vin est si tresbon, aromatique et fort que ung bon buveur n'en pourroit boyre une quarte chascun moys. (Jehan THENAUD, Traité de poesie ou Traité de science poeticque, 1515-1519, p. 142)

22c) Et par ce que c'estoit en temps serain et bien attrempé, son pere luy feist faire des bottes fauves ; Babin les nomme brodequins. (François RABELAIS, Gargantua, 1542, p. 154)

22d) Et par ce que la traitte n'estoit pas trop longue, ilz arriverent de bonne heure au logis : là ou ilz se rafreschirent en beuvant, et beurent en se rafreschissant (Bonaventure DES PÉRIERS, Nouvelles récréations et joyeux devis, 1558, p. 128)

Par ce que avec signification causale lie deux propositions qui n'ont pas de lien ontologique entre elles et qui, sans conjonctions, seraient trop éloignées sémantiquement pour établir une conjonction logique causale.

\subsection{Puisque}

Nous avons déjà montré que le subordonnant temporel de postériorité ou qui a le sens de 'depuis' peut devenir causal. Traugott et König (1991 : 195) ont constaté cette évolution pour plusieurs langues, comme le latin (dum), l'allemand, le finnois, le roumain. En allemand la conjonction denn 'car' ne se distinguait pas de l'adverbe temporel dann 'puis' jusqu'au $18^{\mathrm{e}}$ siècle. Ce n'est qu'à partir de la deuxième moitié du 18 siècle qu'on a fait une distincion artificielle (eine geschichtlich unbegründete, an sich begreifliche, in die volkssprache nicht eingedrungene scheidung, Grimm 1854-1960, II: 740) entre dann, adverbe temporel, et denn, conjonction de coordination. Luther avait utilisé denn dans les deux sens et il avait rejeté dann, tandis que d'autres auteurs de l'époque, 
comme le traducteur Heinrich Steinhöwel (1412-1482) ou l'écrivain Johann Fischart (1546-1591), utilisaient exclusivement dann.

L'adverbe de postériorité a suivi, dans plusieurs langues, un chemin lui permettant d'introduire une proposition. En italien, on trouve la signification de 'par le fait que' déjà chez Boccace (1353) (Cortelazzo/Zolli 2008: 1218), mais jusqu'aujourd'hui, la signification causale peut être liée à des traits temporels. :

23a) Poiché si è fatto tardi rimandiamo tutto il resto a domani.

23b) Lo sapeva, poiché qualcuno l'aveva informato

La proposition causale introduite par poiché peut être antéposée ou postposée dans la phrase, tandis qu'une proposition introduite par siccome se place toujours au début de la phrase. Siccome qui s'utilise dès le $13^{\mathrm{e}}$ siècle comme conjonction causale et qui consiste en une fusion de si 'ainsi' et come 'comme' a suivi le même parcours de changement sémantique que comme en français, ce qui se voit aussi dans sa position antéposée dans la phrase :

24) Siccome vuoi fare di testa tua, pagherai di tasca tua.

À côté de poiché il y a un autre adverbe temporel italien qui se lie avec la conjonction che pour donner un connecteur causal : già + che $>$ giacché. Giacché peut exprimer la causalité, liée à un moment temporel : 'parce que la cause est donnée dans un certain moment la conséquence est inévitable'.

25) Figlio mio, non essere sensuale, giacché la sensualità conduce alla fornicazione ${ }^{5}$

L'évolution du temporel au causal se retrouve dans le cas de la conjonction française puisque, qui est la forme tardivement soudée de l'ancienne locution post que (vers 980), puis que (vers 1080) (Rey 200 : 3006). Son ancien sens temporel de après que' a rapidement décliné au $14^{\mathrm{e}}$ siècle. Il fut supplanté par le sens logique 'étant donné que, du moment que' avec lequel le mot introduit une cause en faisant reconnaitre le rapport de cause à effet comme incontestable.

Le processus de l'effacement de la signification temporelle commença par des emplois dans lesquels puisque avait une valeur à la fois causale et temporelle :

26) Puis qu'ilz eurent failly a la lemproye, le curé mist la table et firent la meilleure chere qu'ilz sceurent. (FEW IX, C.N.N., c.1456-1467, 263)

La majorité des exemples trouvés dans Frantext pour le 12e jusqu'au début du $16^{\mathrm{e}}$ siècle permet une lecture dans le sens temporel véhiculant le sens causal :

\footnotetext{
5 http://www.garganonline.net/Didache2.html
} 
27a) Mes, sire, puis que rois s'amort a croire les mavés larrons et il laisse les haut barons et guepist le chief por la queue, (ANONYME, Roman de Renart, branche I. Jugement de Renart, Siège de Maupertuis, Renart teinturier, 1180, p. 42)

27b) Puis que morir voi feble et fort, / Coument panrrai en moi confort, / Que de mort me puisse deffendre ? N'en voi nul, tant ait grant effort, Que des piez n'ost le contrefort, (RUTEBEUF, Oeuvres complètes, 12491277, p. 336)

27c) Mourons acop, puis que nostre princesse De nous s'esloingne et de nous aymer cesse ! (Jean LEMAIRE DE BELGES, Les Épîtres de l'amant vert, $1511,10)$

27d) [...] si feroie, dit elle, car puis que on y va pour paier, on le treuve à toutes heures. (Philippe de Vigneulles, Les Cent Nouvelles nouvelles, 1515, p. 94)

27e) Donque, puis que vous congnoissez / D'où tout le bien vous peult venir, / Tous faulx remeddes délaissez / Pour au seur et vray vous tenir. (Marguerite de NAVARRE, Le Mallade, 1535, p. 24)

Mais on trouve aussi des exemples dans lesquels puis que exprime un sens purement causal :

28a) Dieu jure et Sainte Patenostre ja mes ne gerra a sa coste : qu'a l'en a faire d'ome en chanbres, puis que il n'a trestoz ses manbres? (ANONYME, Roman de Renart, branche I. Jugement de Renart, Siège de Maupertuis, Renart teinturier, 1180, p. 99)

28b) Je vois, puis que vous le volés. (ADAm Le Bossu dit ADAM De LA HALLE, Le jeu de Robin et Marion, 1285, p. 36)

28c) Et puis que l'oeuvre de fait va devant les affections et les parolles legieres du menu peuple, je me rapporte a toy de conclurre qui est de ce le plus chargié. (Alain CHARTIER Le Quadrilogue invectif, 1422, 39)

28d) Puis que tu te dis empereur, tu ne t'en yras pas sans estre pugny. (ANONYME, Le Violier des histoires rommaines moralisées, 1521, p. 158, 57)

28e) Quoy qu'il en soit, puis que nous avons un Dieu qui peult en un moment sans difficulté retirer les siens du sepulchre, il les pourra bien pourmener au bort tant qu'il vouldra, sans permectre qu'ilz tombent dedans, jusque à ce qu'il en soit temps. (CALVIN Jean, Lettres à Monsieur et Madame de Falais, 1543-1554, p. 63)

Les propositions introduites par puis que avec une signification causale ont un sens plus éloigné de celui de la proposition principale et qui ne s'interprète pas à première vue comme causalité. La locution conjonctive apporte alors le sens causal 
et elle est indispensable à l'interprétation des énoncés en termes de 'causes' et 'conséquences'.

$\mathrm{Au}$ moins depuis le $17^{\mathrm{e}}$ siècle, puisque sert aussi à introduire la cause qui explique non le fait énoncé dans la principale, mais son énonciation.

29a) Et de là il est clair que la nature de l'affirmation est d'unir et d'identifier, pour le dire ainsi, le sujet avec l'attribut, puisque c'est ce qui est signifié par le mot est. (Antoine ARNAULD \& Pierre NiCOLE, La Logique ou l'Art de penser, 1662, p. 198)

29b) Je vous crois, puisque vous le dites. (MARIVAUX, La Double inconstance, 1724 , p. 230

Cela correspond à un plus haut degré de généralisation de sa signification, qui le caractérise comme vrai connecteur causal sans traits temporels.

\section{Conclusions sur la nature de la grammaticalisation}

Si l'on considère la grammaticalisation, il n'y pas de phénomène qui soit exclusivement synchronique ou diachronique, mais la synchronie et la diachronie sont deux perspectives selon lesquelles on examine un fait. La grammaticalisation ne pourrait pas être un procédé de changement diachronique si elle n'était pas une espèce de variation synchronique. C'est ce qu'on a pu voir dans l'exemple des locutions conjonctives transformées en causales qui gardent des traits temporels (puisque) ou qui permettent une interprétation autre que causale comme c'est le cas pour le pronom ce (par ce que).

Cela a des conséquences méthodologiques pour la reconstruction linguistique, dans laquelle nous comparons des formes ou des significations comme si elles existaient synchroniquement. Les relations étant constatées de cette manière, on les dynamise après coup, par exemple en analysant une forme comme plus archaïque que l'autre. On interprète la relation entre deux éléments comme diachronique, en posant qu'un des deux provient de l'autre ou que les deux dérivent d'une troisième forme originaire. Quand on procède de cette manière, on limite notre capacité de reconnaître une relation de grammaticalisation en situation synchronique. Il en résulte que la situation méthodologique du chercheur est complètement différente quand il regarde les données à un stade de la langue et quand il analyse des phénomènes qui appartiennent à différentes couches historiques. S'il y a variation dans cette dernière situation, elle a une direction intrinsèque dans le sens d'une grammaticalisation qui mène obligatoirement de la variante plus ancienne vers la nouvelle. S'il y a variation dans un stade de la langue, cela ne montre pas une relation intrinsèque. C'est pourquoi la dynamisation d'une telle variation est basée sur l'analogie avec le modèle de changements historiquement attestés.

Dans quelle mesure est-il possible de regarder le développement des connecteurs causaux en français en termes de grammaticalisation? Prenons comme base la définition de la grammaticalisation donnée par Christian Lehmann: «Grammaticalization of a linguistic sign is a process in which it loses in autonomy by 
becoming more subject to constraints of the linguistic system » (Lehmann 2004: 153). Cette définition contient plusieurs notions qui demandent une explication, le concept d'« autonomie » et sa perte ainsi que le concept de « contraintes du système linguistique ».

Le concept de grammaticalisation est relativement large quant à son application à un signe linguistique qui peut être simple ou complexe. Mais il est très strict quant à la spécificité du chemin parcouru par le signe linguistique dans sa perte d'autonomie. Un autre chemin est l'intégration dans une unité lexicale par la lexicalisation. Pour prouver qu'un phénomène linguistique subit une grammaticalisation, il faut montrer les faits suivants :

$1^{\circ} \mathrm{Il}$ y a deux stades historiques de la langue L, un L1 plus ancien et un L2 plus récent

$2^{\circ}$ Dans L1, il y a la forme F1 et, dans L2, la forme F2. F2 est diachroniquement identique à $\mathrm{F} 1$.

$3^{\circ} \mathrm{F} 2$ est plus grammaticale que F1.

Nous discuterons ces trois conditions essentielles, tout en les mettant en relation avec les locutions conjonctives et en essayant de clarifier les notions d'« autonomie » et de « contraintes du système linguistique ».

$1^{\circ}$ Des cas de variations dans lesquels les formes F1 et F2 appartiennent au même stade d'une langue ne comptent pas comme preuve empirique pour la grammaticalisation. La notion de «grammaticalisation » comprend l'idée d'une direction de la variation. Des cas de variation dans lesquels F1 est reconstruit de F2 ne comptent pas non plus comme preuve historique. Une reconstruction faite en toute responsabilité implique qu'elle soit compatible avec les tendances du changement diachronique qui s'avèrent être des généralisations à partir de changements historiques attestés. Une de ces tendances est sans doute la grammaticalisation. Alors, la grammaticalisation joue un rôle méthodologique important dans la reconstruction, mais la reconstruction ne peut jouer un rôle illustratif dans la grammaticalisation. Autrement l'utilisation de formes reconstruites comme preuve empirique pour la grammaticalisation risquerait de devenir circulaire.

Les locutions conjonctives par ce que et puis que et les conjonctions parce que et puisque appartiennent à des stades différents du français et nous n'avons pas reconstruit la signification du premier stade, mais nous les avons découvert à partir de textes de l'époque. Dans les locutions conjonctives, nous avons pu constater pour puis que un trait temporel, celui de "postériorité», et, dans par ce que, un pronom qui correspond normalement à une relation factuelle ou existentielle. Ces traits coexistent, pendant un certain temps, avec la signification causale. Avec la fusion des éléments de la locution, cette signification causale se met de plus en plus au premier plan et les éléments de la locution ont perdu partiellement leur autonomie. Ce processus n'est pas encore terminé parce qu'on peut revitaliser la signification première des formes du stade L1, mais cela exige un effort cognitif plus grand ou un contexte spécifique.

$2^{\circ}$ La notion d'« identité diachronique » inclut celle d'« identité », notion élémentaire qui ne peut pas être définie. L'intuition qui se trouve derrière l'identité diachronique' est la suivante: une unité individuelle existe pendant un certain temps et reste identique à elle-même à travers l'usage qui la modifie. Elle garde son identité bien que 
tout aspect de l'unité individuelle puisse changer. On suppose qu'il y a identité diachronique malgré la différence des signifiés et malgré la différence des signifiants.

Pour les locutions conjonctives étudiées, on peut bien constater une identité diachronique avec les conjonctions causales qui se reflète aussi bien dans la forme phonique que dans la continuité des traits sémantiques. Cette identité subsiste malgré le changement de catégorie grammaticale ( $\rightarrow$ conjonction) et la dominance de la valeur causale.

$3^{\circ} \mathrm{La}$ troisième condition présuppose une définition opérationnelle de la grammaticalisation et de ses degrés. Comme les deux stades de la langue ne correspondent pas à la langue contemporaine, le contenu des signes en question peut être difficile à observer. Il est compréhensible que beaucoup d'auteurs se soient appuyés exclusivement sur le côté structurel. Il faut tout de même tenir compte du fait que l'autonomie du signe linguistique et la grammaticalisation sont des notions complexes auxquelles plusieurs facteurs sont corrélés. Si l'on se concentre seulement sur l'un d'eux, le résultat ne sera pas suffisant. La discussion est souvent simplifiée, par exemple, par l'usage de la coalescence (morphème libre $>$ clitique $>$ affixal $>$ fusionnel) comme unique critère de la grammaticalisation.

Dans le cas des locutions conjonctives causales décrites, on peut bien observer une fusion en une forme qui a acquis la fonction nette de subordination causale, mais les traits sémantiques des composantes sont toujours présents et peuvent être activés dans des contextes spécifiques. On peut d'autant plus observer, dans le Moyen Français, que les différentes fonctions des locutions qui correspondent à différents degrés de grammaticalisation se présentent comme une variation.

\section{RÉFÉRENCES BIBLIOGRAPHIQUES}

BAT-ZEEV SHYLDKROT, H., \& KeMMER, S., 1988, « Le développement sémantique des conjonctions en français : quelques concepts généraux », Revue Romane, 23 :1, 9-20.

BERTIN, A., 1997, L'expression de la cause en ancien français, Genève, Droz.

BerTin, A., 2001, "Maintenant: un cas de grammaticalisation? », Langue française, 130, 42-65.

Bybee, J. L., Perkins, R. \& PAgliuCA, W., 1994, The evolution of grammar. Tense, aspect and modality in the languages of the world, Chicago \& London, University of Chicago Press.

COROMINAS, J., 1993, Diccionario crítico etimológico castellano e hispánico, con la colaboración de José A. Pascual. Vol. 4: ME-RE, 1. ed., reimpresión. Madrid, Ed. Gredos, 1993.

Cortelazzo, M., Zolli, P., 2008, Dizionario etimologico della lingua italiana, Bologna, Nicola Zanichelli.

CunHA, A. G. da, 1986, Dicionário etimológico nova fronteira da língua portuguesa. 2.a edição, rev. e acrescida de um supl., 7a impr. Rio de Janeiro, Ed. Nova Fronteira. 
DARDEL (de), R., 1983, Esquisse structurale des subordonnants conjonctionnels en roman commun. Genève, Droz.

Dembrovski, P., F., 1969, Jourdain de Blaye (Jordains de Blaivies), Chanson de geste éditée par P.F.D., Chicago and London, The University of Chicago Press.

FAGARD, B., \& DEGAND, L., 2008, «La fortune des mots : grandeur et décadence de car» in Durand J. Habert B., Laks B. (éds.) Congrès Mondial de Linguistique Française CMLF'08, Paris, Institut de Linguistique Française, 211-223.

GamillscheG, E., 1997, Etymologisches Wörterbuch der französischen Sprache, Heidelberg: Winter.

Grimm, J. et W., 1854-1960, Deutsches Wörterbuch von Jacob Grimm und Wilhelm Grimm, 16 Bde. [in 32 Teilbänden], Leipzig, S. Hirzel.

KLEIN, E., 1979, A comprehensive etymological dictionary of the English language: dealing with the origin of words and their sense development thus illustrating the bistory of civilization and culture, 5. impr., Amsterdam, Elsevier Scientific Publicatons.

KLugE, F., 1999, Etymologisches Wörterbuch der deutschen Sprache, Bearb. Von Elmar Seebold. 23., erw. Aufl., Berlin, de Gruyter.

LANGACKER R. W., 1991, Foundations of Cognitive Grammar, vol. II: Descriptive Application, Stanford, Stanford University Press.

LAZZERI, G., 1954, Antologia dei primi secoli della letteratura italiana. Ristampa 11942, Milano, Ulrico Hoepli.

Le Trésor de la Langue Française informatisé (TLFi) http:/ / atilf.atilf.fr/tlf.htm

LEHMANN, C., 1995, Thoughts on grammaticalization. Unterschleissheim, LINCOM Europa, LINCOM Studies in Theoretical Linguistics, 1.

LEHMANN, C., 2004, "Theory and method in grammaticalization », Zeitschrift für Germanistische Linguistik 32/2, 152-187.

LINSKILL, J., 1937, Saint Léger. Étude de la langue du manuscrit de Clermont-Ferrand, suivie d'une édition critique du texte avec commentaire et glossaire, Paris, E. Droz.

MARCHELlO-NizIA C., 2006, Grammaticalisation et changement linguistique, Bruxelles, de boeck.

MASSÈrA, A. F.,1920, Sonetti burleschi e realistici dei primi due secoli, Bari, Laterza.

MENÉNDEZ PIDAL, R., 1954-1956, Cantar de mio Cid. Texto gramática y vocabulario, Tercera edición, Madrid, Espasa-Calpe.

Moignet, G., 1969, La Chanson de Roland, texte établi d'après le manuscrit d'Oxford, Paris, Bordas.

Moll, F. de B., 1952, Gramática histórica catalana, Madrid, Gredos.

NunES, J. J., 1970, Crestomatia arcaica. Excertos da literatura portuguesa desde o que mais antigo se conbece até ao século XVI, $7^{\mathrm{a}}$ edição, Lisboa, Teixeira. 
OnIONS, C. T., 1974, The Oxford dictionary of English etymology. Oxford [u.a.], Oxford Univ. Press.

PAR, A., 1923, Sintaxi catalana segons los escrits en prosa de Bernat Metge (1398), Halle, Niemeyer (= ZRPh, Beiheft 66).

Pasch, R., Braube U., Breindl E. \& Waßner U.H., 2003, Handbuch der deutschen Konnektoren. Linguistische Grundlagen der Beschreibung und syntaktische Merkmale der deutschen Satzverknüpfer (Konjunktionen, Satzadverbien und Partikeln), Berlin- New York, Walter de Gruyter.

PFEIFER, W. et al., 1989, Etymologisches Wörterbuch des Deutsche, 3 Bde, Berlin, AkademieVerlag.

RAIBLE, W., 1992, Junktion. Eine Dimension der Sprache und ibre Realisierungsformen zwischen Aggregation und Integration. Heidelberg, C. Winter (Sitzungsberichte der Heidelberger Akademie der Wissenschaften).

REY, A., (dir.), 2000, Dictionnaire historique de la langue française, Paris, Dictionnaires Le Robert.

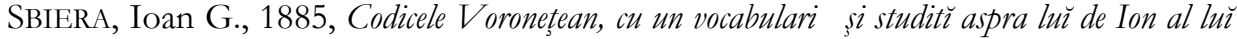
G.S. Cernauț: Edițiunea Academieř Romîne.

SCHMidhauser, B., 1995, Kausalität als linguistische Kategorie: Mittel und Möglichkeiten für Begründungen, Tübingen, Niemeyer.

TraugotT, E. C., König, E., 1991, «The semantics-pragmatics of grammaticalization revisited» in Traugott, E. \& Heine, B. (eds.) Approaches to Grammaticalization, Amsterdam, Benjamins, p.189-218.

WollenberG, J., 1868, «L'évangile selon Jean en vieux provençal». Programme d'invitation à l'examen public du Collège royal français, fixé au 25 septembre 1868, Berlin, s.n., p.1-29.

\section{Bases de données et ressources informatisées}

Base Textuelle Frantext http:/ / atilf.atilf.fr/ frantext.htm

Le Trésor de la Langue Française informatisé: (TLFi) http:/ / atilf.atilf.fr/tlf.htm 